\title{
Study of the phenomenology of the hadronic interactions in the reconstruction of extensive air showers
}

\section{R. Wiklich Sobrinho*广}

Center for Natural Sciences and Humanities, Federal University of ABC, Santo André, Brazil. E-mail: rafaelawiklichs@gmail.com

\section{A. Leigui de Oliveria}

Center for Natural Sciences and Humanities, Federal University of ABC, Santo André, Brazil.

E-mail: leiguieufabc.edu.br

We present a study of the average longitudinal profile for extensive air showers produced using CORSIKA (COsmic Ray SImulations for KAscade) code considering different energies and compositions. Several models for hadronic interactions at ultra-high energies, such as EPOS-LHC, QGSJetII-04, and SIBYLL2.3c, are used to simulate the particle production and their distribution in the atmosphere. We show a method to measure the $\sigma_{p-a i r}$ and attenuation length for air shower simulated data.

36th International Cosmic Ray Conference -ICRC2019-

July 24th - August 1st, 2019

Madison, WI, U.S.A.

* Speaker.

${ }^{\dagger}$ UFABC - Federal University of ABC 


\section{Introduction}

The Ultra-High Energy Cosmic Rays (UHECRs) are the most energetic particles already observed, demanding huge area experiments to their detection due to a very low flux [1]. Using indirect detection techniques to measure the Extensive Air Showers (EASs), composed by the secondary particles developed in the atmosphere, it is possible to infer the primary cosmic ray information, such as energy, arrival direction, and mass composition.

The detailed study of extensive air showers allows us to understand the hadronic interaction properties of the particles and to restrict parameters of the current hadronic interaction models. The proton-air cross-section of cosmic rays is estimated using data collected by large cosmic ray experiments, at energies far beyond what particle accelerators can produce at all. It is still not possible to calculate the proton-air cross-section value at that higher energies with quantum chromodynamics theory [2]. Therefore particle collision measurements at high and ultra-high energy are of great interest to explore a new physics.

In this work, the phenomenological study proposed is based on the use of a function capable of providing the proton-air cross-section from the attenuation length using Monte Carlo EAS simulations in order to estimate the proton-air cross-section from cosmic ray experiment real data. In addition, we present a correlational study of some observables as a function of the depth of air shower maximum, denoted $X_{\max }$.

\section{Simulations and first results}

To perform the presented study, we simulated a library of EASs, using the software CORSIKA version 76900 [3]. We considered sets of 1000 showers initiated by cosmic primaries of $\mathrm{p}, \mathrm{He}, \mathrm{N}$, $\mathrm{Si}, \mathrm{Fe}$, in the energy range from $10^{14}$ to $10^{19.5} \mathrm{eV}$, at zenith angles from 0 to 60 degrees. It was considered three hadronic interaction models: EPOS (LHC) [4], QGSJETII-04 [5], and SIBYLL 2.3c [6]. This set of simulations was used to study three aspects, the first one concerns the average number of muons produced by these EASs, the second was about the correlation of hadronic interaction parameters like elasticity, multiplicity, or ratio of charged pions with the $X_{\max }$, and finally, the third was on the determination of the proton-air cross-section value.

In an initial analysis, we investigated the behavior of the mean number of muons as a function of the energy of the primary particle, selecting the simulations performed only for proton and iron primaries. Therefore, it was verified that the average number of muons increases exponentially with energy (figure 1) and, comparing the same energy range, the average number of muons is higher for iron primaries than protons. Such behavior is expected since heavy nuclei can be considered as the sum of many individual nucleons. In addition, the study of the elongation ratio it was carried out, showing the behavior of the $\left\langle X_{\max }\right\rangle$ as a function of the primary energy in order to verify the performed simulations. In this last result, for the same energy value, the $\left\langle X_{\max }\right\rangle$ for proton primaries is larger than for iron primaries, corroborating with the expected in theory, in which heavier nuclei have higher cross-sections, interacting with the atmosphere as it crosses smaller distances.

On the other hand, we performed a Pearson correlation study to verify the correlation strength between the shower observables and the $X_{\max }$. In all cases, we found a weak anti-correlation, as 


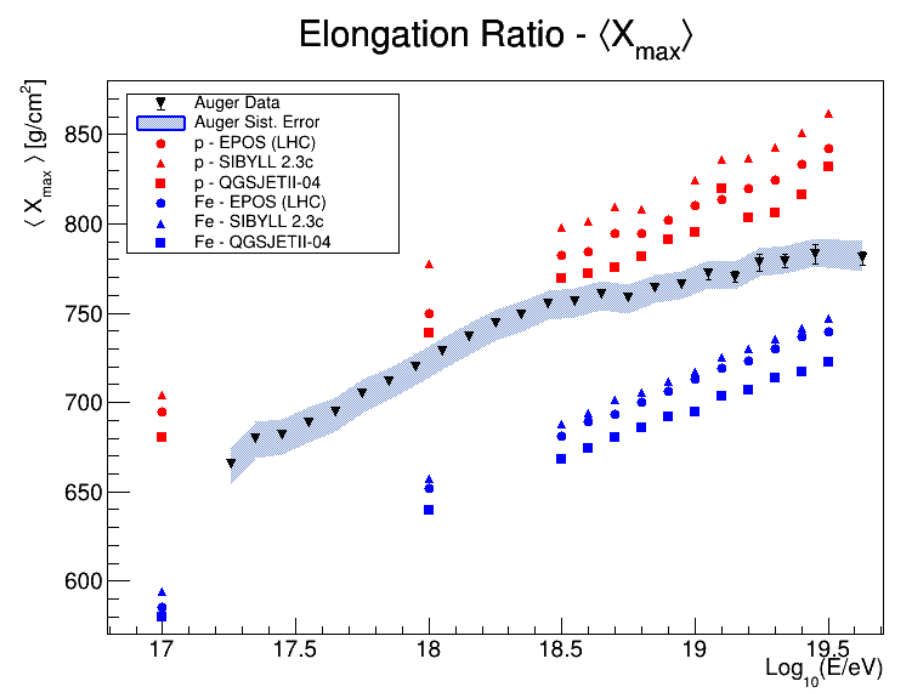

Mean Muon number

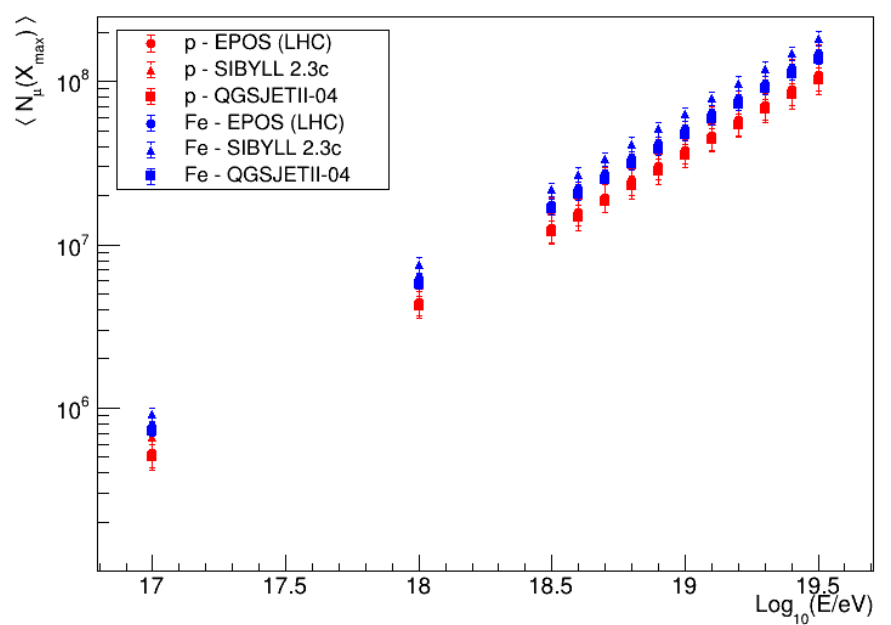

Figure 1: The upper figure shows the elongation rate, red dots indicate proton and blue dots iron simulation. The black dots are data from Pierre Auger Observatory events [7]. The bottom figure shows the average muon number at the $\mathrm{X}_{\max }$ depth.

we can see in one of the results of figure 2. In the future, the purpose of this work is to modify these observables and verify if this correlation changes to verify if the average number of muons increases or decreases with these modifications. 

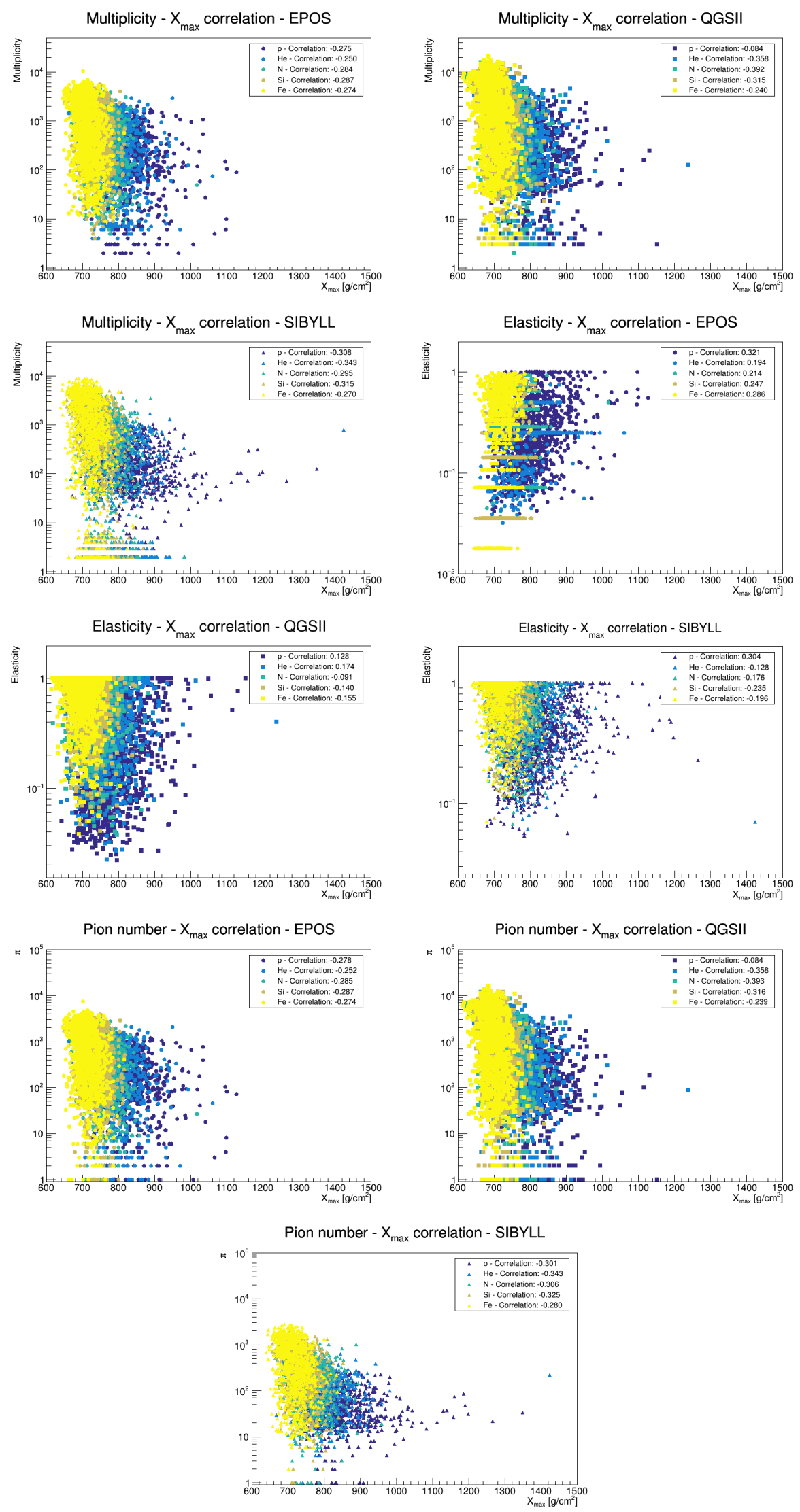

Figure 2: Correlation of first hadronic interaction parameters, multiplicity, elasticity, and ratio of charged pions, with $X_{\max }$ for EPOS(LHC), QGSJetII-04, and \$IBYLL models. 


\section{Determination of $\sigma_{p-a i r}$ and attenuation length for Monte Carlo air shower simulations}

In this paper, we show the determination of the proton-air cross-section using the simulated data. The value $\sigma_{p \text {-air }}$ from the simulation is obtained of particle first hadronic interaction on CORSIKA output data. In the case of experimental data, we can determine the value of $\sigma_{p \text {-air }}$ using the K-factor method [8], for data selection, however, we need first to estimate the conversion of the attenuation length in the proton-air cross-section for the simulated data. The relation between attenuation length and cross-section is given by:

$$
\Lambda_{\eta}=K \times 14.45 m_{p} / \sigma_{p-a i r}^{\text {inel }}
$$

with $14.45 m_{p}=24160 \mathrm{mb} \mathrm{g} \mathrm{cm}^{-2}$ [9], and $\mathrm{K}$ is a constant.

From the previous relation, it is possible to convert the attenuation length of Monte Carlo air showers simulations $\Lambda_{\eta}^{M C}$ in proton-air cross-section. Another very interesting expression is the attenuation length $\left(\Lambda_{\eta}\right)$ that can be calculated from the $X_{\max }$ distribution [2] for given energy:

$$
\begin{gathered}
\log L=\log p\left(X_{\max , i} ; \Lambda_{\eta}\right) \\
p\left(X_{\max , i} ; \Lambda_{\eta}\right)=\left[\Lambda_{\eta}\left(e^{-X_{\eta, \text { start }} / \Lambda_{\eta}}-e^{-X_{\eta, \text { end }} / \Lambda_{\eta}}\right)\right]^{-1} e^{-X_{\max } / \Lambda_{\eta}} .
\end{gathered}
$$

The result of the unbinned log-likelihood fit provides the attenuation length. For each simulation, we selected the distribution tail so as to contain data between the ranges from 700 to 1100 $\mathrm{g} / \mathrm{cm}^{2}$. The fit was done in the tail of the distribution since it would select the most penetrating primaries particles of the simulation. This procedure was performed for all simulated energy ranges with some results summarized in table 1 , with statistical errors. In figure 3 , it is possible to verify one of the results of the unbinned log-likelihood fit, considering a fixed energy range for hadronic interaction models EPOS, QGSJetII-04, and SIBYLL2.3c. 

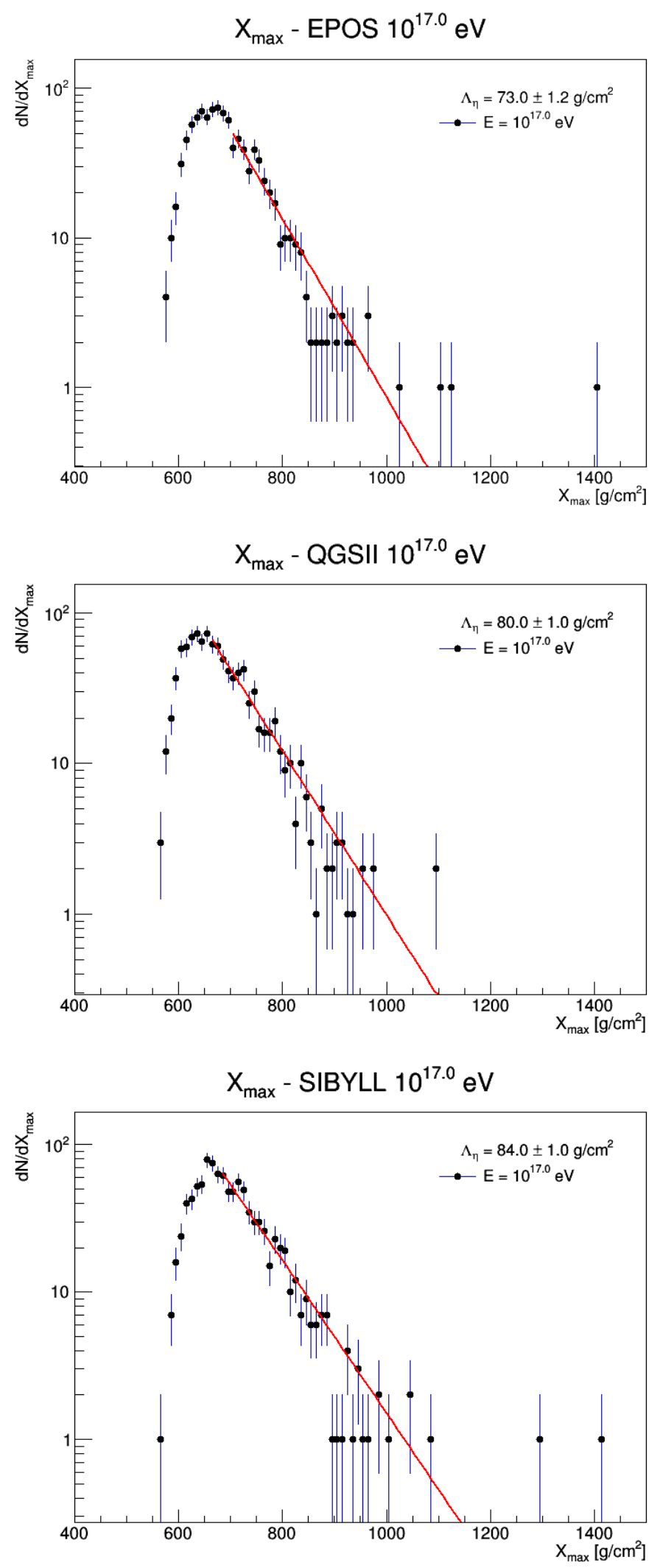

Figure 3: The $X_{\max }$ distributions. The result of the unbinned log-likelihood fit providing the attenuation length. In this figure it was considered primary particles with of $10^{17} \mathrm{eV}$, for EPOS, QGSJetII-04 and SIBYLL models. 


\section{1 $\Lambda_{\eta}^{M C}$ to $\sigma_{p-a i r}$ conversion}

Given the values of the attenuation length for all the energy ranges and identifying the value of the proton-air cross-section for each of these energies, it is possible to use equation 3.1, that connects these two parameters. By performing a fit using this function, we can estimate the value of the proton-air cross-section with its respective statistical error for different values of attenuation length. In figure 4, we show the result of this conversion.

On the other hand, it is possible to use experimental data and reproduce the analysis of the previous item. Thus, we could estimate the attenuation length of cosmic ray experiment data and, using the conversion function $\Lambda_{\eta}^{M C}$ to $\sigma_{p-a i r}$, estimate the proton-air cross-section of these experimental data.
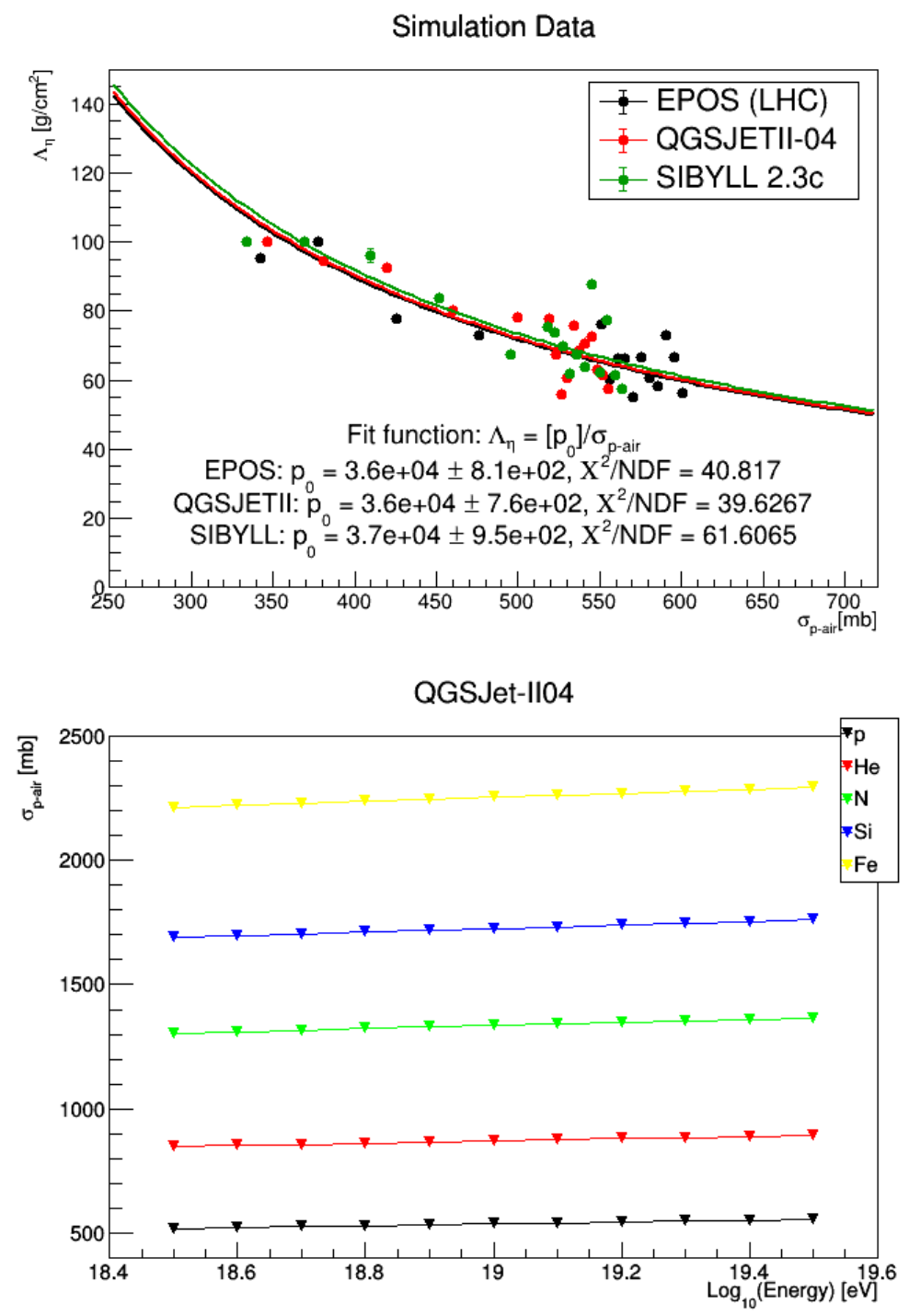

Figure 4: Conversion of $\Lambda_{\eta}$ to $\sigma_{p \text {-air. }}$ The solid lines shows the $\Lambda_{\eta}$ measurement and its projection to the proton-air cross-section for different models. The bottom figure shows the proton-air cross-section for QGSJetII-04 model. 


\begin{tabular}{|l||l||l||l||l||l||l|}
\hline \multicolumn{7}{|c|}{ Determination of $\Lambda_{\eta}$ for the hadronic interaction models } \\
\hline Models & $10^{14} \mathrm{eV}$ & $10^{15} \mathrm{eV}$ & $10^{16} \mathrm{eV}$ & $10^{17} \mathrm{eV}$ & $10^{18} \mathrm{eV}$ & $10^{19} \mathrm{eV}$ \\
\hline QGSJetII-04 & $100.0 \pm 0.6$ & $94.7 \pm 1.4$ & $92.5 \pm 1.0$ & $80.0 \pm 1.0$ & $78.2 \pm 1.1$ & $68.5 \pm 0.4$ \\
EPOS-LHC & $95.2 \pm 1.5$ & $99.1 .0 \pm 0.1$ & $77.6 \pm 0.9$ & $73.0 \pm 1.2$ & $69.1 \pm 0.8$ & $66.6 \pm 0.7$ \\
SIBYLL2.3c & $100.1 \pm 0.1$ & $100.0 \pm 0.1$ & $96.3 \pm 2.0$ & $84.0 \pm 1.0$ & $67.6 \pm 1.0$ & $64.0 \pm 0.7$ \\
\hline
\end{tabular}

Table 1: Some results of determination of $\Lambda_{\eta}$ using Monte Carlo simulations.

\section{Conclusions}

It was presented the study of the EASs parameters through simulations with the CORSIKA software. The presented $\sigma_{p-a i r}$ analysis is the method used to compare the simulation data with results obtained in cosmic ray experiments, showing a strong model dependence on the interpretation of air shower simulation and data. This work is in progress and the results obtained will be used for reconstruction analysis of EASs data acquired by UHECRs observatories.

\section{Acknowledgements}

We are very grateful to Vitor Prestes and Carlos J. Todero for the help and many discussions about this work. This work was supported by Conselho Nacional de Desenvolvimento Científico e Tecnológico - CNPq (Proc. 167920/2017-0).

\section{References}

[1] Mollerach, S., \& Roulet, E. (2018). Progress in high-energy cosmic ray physics. Progress in Particle and Nuclear Physics, 98, 85-118.

[2] Ulrich, Ralf. "Extension of the measurement of the proton-air cross section with the Pierre Auger Observatory." PoS (2015): 401.

[3] D. Heck et al., Report FZKA 6019 (1998).

[4] T. Pierog and K. Werner, Nucl. Phys. B - Proc. Suppl. 196 (2009) 102.

[5] S. Ostapchenko, Phys. Rev. D 83 (2011) 014018.

[6] E. Ahn et al., Phys. Rev. D 80 (2009) 094003.

[7] Pierre Auger Collaboration, A. Aab et al.Phys. Rev.D90(2014) 122005, [1409.4809].

[8] Hara, T., et al. Phys. Rev. Lett. 50.26 (1983): 2058.

[9] Ulrich, R., et al., New J. Phys.11, 065018 (2009). 